\title{
Brain-Derived Neurotrophic Factor Expands Ocular Dominance Columns in Visual Cortex in Monocularly Deprived and Nondeprived Kittens But Does Not in Adult Cats
}

\author{
Yoshio Hata, ${ }^{1,2}$ Minoru Ohshima, ${ }^{1,2}$ Satoshi Ichisaka, ${ }^{1,2}$ Masumi Wakita, ${ }^{2}$ Mitsuhiro Fukuda, ${ }^{2}$ and \\ Tadaharu Tsumoto ${ }^{1,2}$ \\ ${ }^{1}$ CREST, Japan Science and Technology Corporation, and 2Department of Neurophysiology, Biomedical Research \\ Center, Osaka University Medical School, Osaka 565-0871, Japan
}

\begin{abstract}
Segregation and stabilization of thalamocortical afferents to eye-specific patches, so-called "ocular dominance (OD) columns," in visual cortex are hypothesized to be based on activity-dependent competition for trophic factors such as brain-derived neurotrophic factor (BDNF) between afferents representing the two eyes during the critical period of postnatal development. To test this hypothesis we observed effects of an intracortical infusion of BDNF on OD columns in monocularly deprived kittens and also compared effects between normal
\end{abstract}

kittens and adult cats. BDNF had a hypertrophic action on afferents irrespective of visual inputs so that it desegregated OD columns in the visual cortex of deprived and normal kittens, but this action was not seen in the adults, substantiating its hypothesized trophic role in plasticity of OD columns in the developing visual cortex.

Key words: ocular dominance columns; brain-derived neurotrophic factor; monocular deprivation; development; plasticity; visual cortex; cat
The formation of ocular dominance (OD) columns in the primary visual cortex of carnivores and primates is one of the best-studied examples of plasticity of neural circuits in the developing brain. Segregation of afferents from lateral geniculate nucleus (LGN) into eye-specific patches is based on activity-dependent competition between afferents representing the different eyes during the critical period of postnatal development (Hubel and Wiesel, 1970; Stryker and Harris, 1986; Shatz, 1990). For example, a reduction of visual inputs from one eye by lid suture leads to a shrinkage of columns representing the closed eye and an expansion of columns representing the open eye (Shatz and Stryker, 1978; LeVay et al., 1980). Regarding a mechanism of such a change, it is hypothesized that afferents from each eye compete for trophic factors whose production and/or release is regulated by neuronal activity (Purves, 1988). Neurotrophins such as brain-derived neurotrophic factor (BDNF) are suggested to be trophic molecules necessary for formation and/or stabilization of OD columns (Singer, 1995; Thoenen, 1995; Cellerino and Maffei, 1996; Katz and Shatz, 1996; McAllister et al., 1999). For example, it was reported that an injection of either BDNF or another neurotrophin, NT-4/5, or an inhibitor of receptors for these neurotrophins into the visual cortex of normal kittens without visual deprivation interferes with the formation of OD columns (Cabelli et al., 1995, 1997).

These results raise the following questions: If BDNF plays such a role as a trophic factor in the formation and/or stabilization of OD columns, does an infusion of BDNF in the visual cortex of monocularly deprived (MD) kittens prevent a shrinkage of OD

Received Oct. 12, 1999; revised Nov. 15, 1999; accepted Nov. 17, 1999.

This study was supported by Grant-in-Aid 07279102 from the Monbusho to T.T. We thank Sumitomo Pharmaceutical Co., Ltd., for gifts of recombinant BDNF. We also thank Drs. Hiroshi Hatanaka and Michael P. Stryker for helpf ul comments.

Correspondence should be addressed to Dr. Yoshio Hata, Department of Neurophysiology, Biomedical Research Center, Osaka University Medical School, 2-2 Yamadaoka, Suita, Osaka 565-0871, Japan. E-mail: hata@nphys.med.osaka-u.ac.jp. Copyright (C) 2000 Society for Neuroscience $\quad 0270-6474 / 00 / 200001-\bullet \$ 15.00 / 0$ columns corresponding to the deprived eye? Is such an action of BDNF, if it exists, seen specifically during the critical period when synaptic competition for trophic factors is supposed to operate? It is rather surprising that these questions have not been addressed previously except for a brief report (Galuske et al., 1996) and an abstract (Gillespie et al., 1996), which showed that an infusion of BDNF or NT-4/5 in the cortex modified the monocular deprivation-induced change in eye preference of cortical neurons. In these studies, however, visual responses of cortical neurons were observed with electrophysiological and optical recording techniques, and thus the results might reflect a modification of intracortical network rather than geniculocortical afferents that make OD columns.

In the present study, we used anatomical techniques to examine effects of the infusion of BDNF on OD columns representing each eye in MD kittens and also compared effects of BDNF on the OD columns between kittens and adult cats. We found that BDNF had a hypertrophic action on LGN afferents irrespective of visual inputs so that it desegregated OD columns in the cortex of deprived and nondeprived kittens, but this action was not seen in the adults.

Parts of the data were published previously in abstract form (Hata et al., 1996).

This article is published in The Journal of Neuroscience, Rapid Communications Section, which publishes brief, peerreviewed papers online, not in print. Rapid Communications are posted online approximately one month earlier than they would appear if printed. They are listed in the Table of Contents of the next open issue of JNeurosci. Cite this article as: JNeurosci, 2000, 20:RC57 (1-5). The publication date is the date of posting online at www.jneurosci.org.

http://www.jneurosci.org/cgi/content/full/3880 


\section{MATERIALS AND METHODS}

Surgery. All kittens in the present study were born in the breeding colony of Osaka University Medical School, and the experimental procedures met the regulation of the Animal Care Committee of Osaka University Medical School. Under anesthesia with $\mathrm{N}_{2} \mathrm{O}: \mathrm{O}_{2}$ (2:1) combined with isoflurane $(1.5-3.5 \%)$ a $30 \mathrm{G}$ stainless steel cannula connected to an osmotic minipump (Alzet 2002; Alza, Palo Alto, CA) was implanted in the primary visual cortex (stereotaxic location: anterior, -2.0 ; lateral, 2.0; depth from cortical surface, $2.0 \mathrm{~mm}$ ) in kittens at postnatal days 35-38 and in adult cats (see Table 1). Visual cortex was infused with BDNF solution continuously at a rate of $6 \mu \mathrm{g} / \mathrm{d}(0.5 \mathrm{mg} / \mathrm{ml}$ in PBS containing $0.1 \% \mathrm{BSA}, 0.5 \mu \mathrm{l} / \mathrm{hr}$, for 2 weeks), which is reported to affect physiological plasticity of the cortex after monocular deprivation (Galuske et al., 1996). The other hemisphere was infused with cytochrome $c$ solution $(0.5 \mathrm{mg} / \mathrm{ml}$ in the same vehicle $)$ as control for nonspecific effects of the protein infusion. Two days after implantation, one eye was deprived of vision by eyelid suture. For anatomical demonstration of the geniculocortical afferent termination, we injected $\left[{ }^{3} \mathrm{H}\right]$ proline $(1.7-2.1$ $\mathrm{mCi}$ in $20 \mu \mathrm{l}$ of saline) into one eye $10-13 \mathrm{~d}$ before the terminal perfusion. All the surgical procedures were performed using sterile procedures under the same anesthetic condition as mentioned above. All of incisions were infiltrated with xylocaine. Animals were treated with an antimicrobial agent (enrofloxacin, $5 \mathrm{mg} / \mathrm{kg}$ ) every day after the surgery until euthanasia.

Histology. At the day of termination, the animals were killed with an overdose of Nembutal (100 mg/kg, i.v.) and perfused transcardially with saline followed by $2 \%$ glutaraldehyde in $0.1 \mathrm{M}$ phosphate buffer (PB). The caudal part of the cortex, which includes the primary visual cortex, was unfolded and flattened between two glass slides and then post-fixed in $4 \%$ paraformaldehyde and $30 \%$ sucrose in $\mathrm{PB}$, as described previously (Hata and Stryker, 1994). The flattened cortex was then sectioned tangentially (thickness, $40 \mu \mathrm{m}$ ) on the frozen microtome. Several animals used for immunohistochemistry were perfused with saline, followed by $4 \%$ paraformaldehyde, and tissue blocks containing visual cortex were post-fixed in $4 \%$ paraformaldehyde and 30\% sucrose in PB. The blocks were sectioned on the frozen microtome in the horizontal plane (thickness, $30 \mu \mathrm{m}$ ).

Transneuronal labeling. For autoradiography, sections were mounted onto gelatinized slides, defatted in xylene, and covered with autoradiographic emulsion (NTB-2; Eastman Kodak, Rochester, NY). After exposure for $4-8$ weeks, photographs of the dark field image were taken and scanned for further processing on the computer. Photomontages of labeling in layer IV were made from images of several sections with the aid of image-processing software (Photoshop; Adobe, Mountain View,
CA). Because the intensity of labeling was not necessarily uniform, and it was necessary to adjust contrasts of labeled OD patches in different regions to match one another to make the photomontages, one cannot infer the absolute intensity of labeling from photomontages presented in this paper. However, the montages do represent the areas of OD patches accurately. The size of OD was measured as proportion of the cortical area of V1 occupied by labeled afferents. Measurements were made on binary images of OD column obtained by delineating the border of labeled area on the autoradiography by hand. Circular areas with diameter of $>2.5 \mathrm{~mm}$ were selected for measurements at the location including the BDNF injection site ("near" site) and at the distance of $>4.0 \mathrm{~mm}$ anterior to the injection site ("far" site). In the case of horizontal sections, areas that contained the labeling in cortical layer IV were selected for column size measurement.

Immunohistochemistry. For demonstration of the cortical region infused with BDNF, sections were processed for standard indirect immunohistochemistry with anti-BDNF antibody. Briefly, sections were incubated overnight at $4^{\circ} \mathrm{C}$ in a blocking solution composed of $5 \%$ skim milk (Difco, Detroit, MI) and $0.3 \%$ Triton X-100 in PB. They were then transferred into a solution of $1 \%$ skim milk, $2 \%$ normal horse serum and $0.3 \%$ Triton $\mathrm{X}-100$ containing the primary antibody (rabbit anti-BDNF polyclonal antibody; Chemicon, Temecula, CA) at a dilution of 1:1000 and kept at $4^{\circ} \mathrm{C}$ for $72 \mathrm{hr}$. After three washes in $\mathrm{PB}$, sections were incubated overnight at $4^{\circ} \mathrm{C}$ in a solution containing rabbit biotinylated secondary antibody (Vector Laboratories, Burlingame, CA) in $1 \%$ skim milk, $2 \%$ normal horse serum, and $0.3 \%$ Triton X-100. After three washes in PB, the sections were transferred into an avidin-HRP complex (Vector) for 3-4 hr, washed for at least $1 \mathrm{hr}$, and finally reacted with a solution of $0.05 \%$ diaminobenzidine hydrochloride, $0.25 \%$ nickel sulfate, and $0.01 \%$ of hydrogen peroxide. All sections were mounted on gelatinized slides, dehydrated in graded series of ethyl alcohol, cleared in xylene, and coverslipped.

\section{RESULTS}

The present data were obtained from nine kittens and four adult cats (older than 2 years of age), and hemispheres in which transneuronal labeling was successful were chosen for analysis, as listed in Table 1. In all the kittens the intracortical infusion of BDNF was initiated at 5 weeks of age (postnatal days 35-38). At this age, an adult-like patchy pattern of geniculocortical afferents has largely been formed in layer IV of the visual cortex (LeVay et al., 1978). Therefore, the present results show the effects of

Table 1. Experimental protocol of each animal

\begin{tabular}{|c|c|c|c|c|c|c|c|}
\hline \multirow[b]{2}{*}{ ID } & \multicolumn{2}{|c|}{ Cannula implant in cortex } & \multicolumn{2}{|c|}{ Eyelid suture } & \multicolumn{2}{|c|}{${ }^{3} \mathrm{H}$ injection into eye } & \multirow[b]{2}{*}{ Termination } \\
\hline & Age & Side & Age & Side & Age & Side & \\
\hline \multicolumn{8}{|c|}{ Kittens, monocular deprivation } \\
\hline YH8 & PD 35 & $\begin{array}{l}\mathrm{L}(\mathrm{BDNF}) \\
\mathrm{R}(\mathrm{CytC})\end{array}$ & PD 37 & $\mathrm{~L}$ & PD 39 & $\mathrm{R}$ & PD 50 \\
\hline YH9 & 37 & $\mathrm{~L}(\mathrm{BDNF})$ & 39 & $\mathrm{~L}$ & 39 & $\mathrm{~L}$ & 51 \\
\hline YH10 & 36 & $\mathrm{~L}(\mathrm{BDNF})$ & 38 & $\mathrm{R}$ & 40 & $\mathrm{~L}$ & 50 \\
\hline YH11 & 38 & $\mathrm{R}(\mathrm{CytC})$ & 40 & $\mathrm{R}$ & 40 & $\mathrm{R}$ & 52 \\
\hline YH50 & 36 & $\mathrm{R}(\mathrm{BDNF})$ & 38 & $\mathrm{R}$ & 38 & $\mathrm{R}$ & 49 \\
\hline YH51 & 38 & $\begin{array}{l}\mathrm{R}(\mathrm{BDNF}) \\
\mathrm{L}(\mathrm{CytC})\end{array}$ & 40 & $\mathrm{R}$ & 40 & $\mathrm{R}$ & 50 \\
\hline \multicolumn{8}{|c|}{ Kittens, no deprivation } \\
\hline YH22 & 38 & $\begin{array}{l}\mathrm{L}(\mathrm{BDNF}) \\
\mathrm{R}(\mathrm{CytC})\end{array}$ & & & 42 & $\mathrm{R}$ & 52 \\
\hline YH23 & 38 & $\mathrm{~L}(\mathrm{BDNF})$ & & & 42 & $\mathrm{~L}$ & 52 \\
\hline YH29 & 36 & $\mathrm{~L}(\mathrm{BDNF})$ & & & 39 & $\mathrm{~L}$ & 50 \\
\hline \multicolumn{8}{|c|}{ Adults, no deprivation } \\
\hline YH18 & Adult & $\mathrm{L}(\mathrm{BDNF})$ & & & PI 2 & $\mathrm{R}$ & PI 15 \\
\hline YH21 & Adult & $\mathrm{L}(\mathrm{BDNF})$ & & & 2 & $\mathrm{~L}$ & 14 \\
\hline SI1 & Adult & $\mathrm{L}(\mathrm{BDNF})$ & & & 2 & $\mathrm{~L}$ & 14 \\
\hline SI4 & Adult & $\mathrm{L}(\mathrm{BDNF})$ & & & 2 & $\mathrm{R}$ & 14 \\
\hline
\end{tabular}

In kittens, PD indicates postnatal days. In adults, PI indicates postimplantation days. 


\section{Monocularly deprived kitten}

Non-deprived eye column

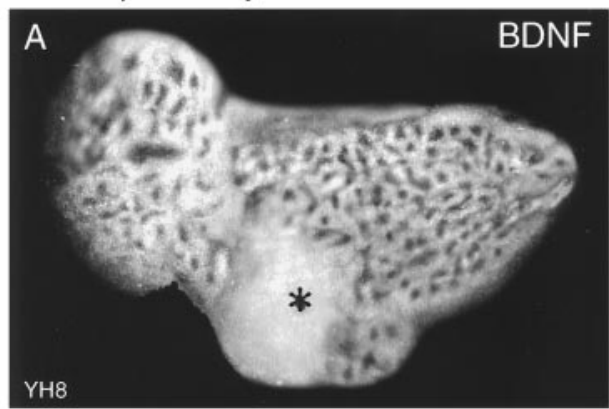

Deprived eye column

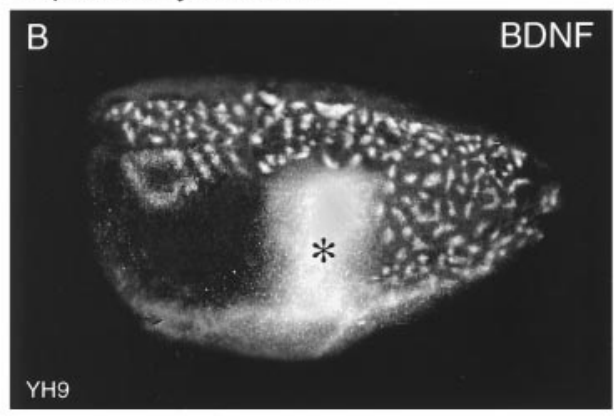

\section{Normal kitten}
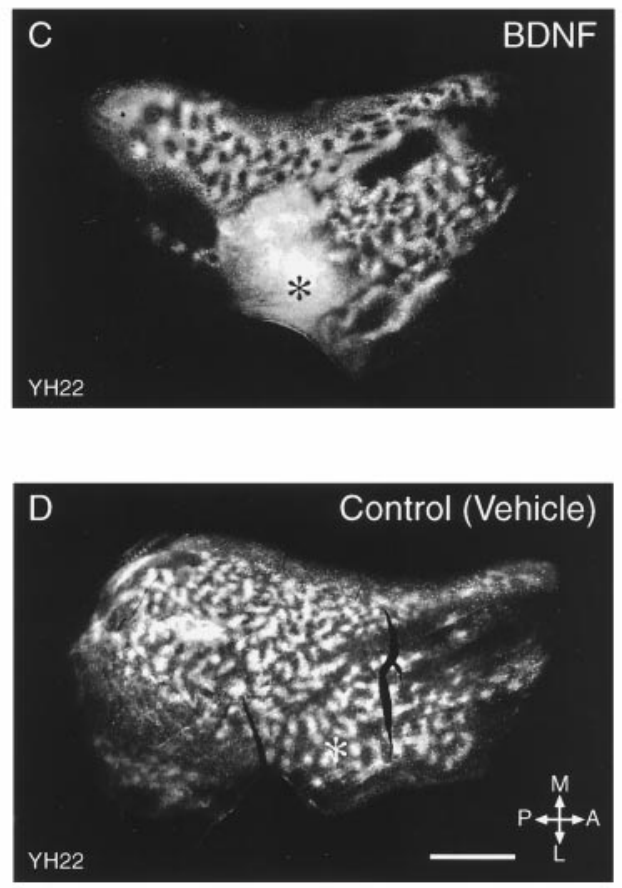

Figure 1. Ocular dominance patches in BDNF-treated and control cortex. Cortical territory innervated by geniculocortical afferents serving one eye was labeled with transneuronal tracer $\left[{ }^{3} \mathrm{H}\right]$ proline. Photomontages of labeling in layer IV were made from sections of flattened visual cortex. Identifiers of the animals are indicated at bottom left. The bright area indicates autoradiographic label. Scale bar in $D, 5 \mathrm{~mm}$ (applies to $A-D)$. $A, B$, Afferents serving the open and closed eyes were labeled, respectively, in monocularly deprived kittens. The cortex was perfused with BDNF, as described in Materials and Methods. $C$, $D$, The visual cortex was perfused with BDNF and vehicle solution, respectively, in a normal kitten. In $D$, the bright area in the top left part of the tissue represents the optic disk. In $B-D$, large black areas without labeling are regions outside layer IV of the cortex. exogenously applied BDNF on the stabilization and refinement of OD columns.

\section{Effects of BDNF on OD columns in monocularly deprived kittens}

In five kittens one eye was closed by eyelid suture $2 \mathrm{~d}$ after starting the infusion of BDNF in the primary visual cortex (Table 1). Subsequently, $\left[{ }^{3} \mathrm{H}\right]$ proline was injected into the deprived or nondeprived eye to label geniculocortical afferent terminals in the cortex. After 2 weeks of the BDNF infusion, the animals were perfused with fixatives, and the cortical tissue was processed to visualize OD columns by conventional autoradiography. The intraocular injection of $\left[{ }^{3} \mathrm{H}\right]$ proline yielded clear transneuronal labeling in layer IV of the primary visual cortex in both BDNFtreated and control hemispheres. The pattern of labeling was, however, quite different between these two hemispheres. Figure $1 \mathrm{~A}$ shows a photomontage of labeling in layer IV of the cortex of a kitten whose nondeprived eye was injected with $\left[{ }^{3} \mathrm{H}\right]$ proline. Except for a region around the infusion site (asterisk), the labeled area (white area) dominated the cortex with tiny holes of unlabeled gap. This domination of labeling reflected an expansion of the cortical territory within which geniculocortical afferents serving the nondeprived eye terminated. It is to be noted that the labeling in the region around the BDNF infusion site showed an almost uniform pattern so that the clear periodic fluctuation of density was not seen, although a faint fluctuation was distinguishable. This indicates that the expansion of afferents representing the nondeprived eye was further enhanced in the BDNF-treated region of the cortex.

On the other hand, OD columns reflecting the deprived eye had a small patchy distribution in the region far from the BDNF infusion site (asterisk), showing a shrinkage of deprived eye columns (Fig. 1B). In the region near the infusion site, however, labeling (white area) was uniform and did not show a sign of patchy distribution, as in the case of nondeprived eye labeling.
This indicates that afferent terminals of the deprived eye expanded rather than shrank in the area in which exogenous BDNF was supplied. Such an expansion of afferent terminals from both deprived and nondeprived eyes in the BDNF-infused area suggests that inputs from the two eyes were no longer segregated into columns but, rather, became intermingled under the action of exogenous BDNF.

\section{Effects of BDNF on OD columns in normal kittens}

The results obtained in the MD kittens suggest that exogenously applied BDNF expands OD columns that have already been formed, and this action is independent of visual deprivation. To confirm that the effect of BDNF is not directly related to monocular deprivation, the next series of experiments were performed in three kittens with normal vision (Table 1). Figure 1C shows an example of flattened cortex of a kitten in which $\left[{ }^{3} \mathrm{H}\right]$ proline was injected into the contralateral eye. Similarly to the observation in the MD kittens, the labeling was nearly uniform in the cortical region around the BDNF infusion site (asterisk), indicating the expansion of cortical territory for geniculocortical afferents representing the injected eye. In this figure a faint fluctuation of labeling density was seen especially at the peripheral region of desegregation, suggesting that the desegregation was not complete in this region. In the other hemisphere of the same animal, such an expansion of OD column was not seen in the area into which the vehicle solution was injected, and the entire region showed a periodic fluctuation of the intensity of labeling (Fig. 1D). Therefore, it seems reasonable to conclude that BDNF has an expanding action on OD columns regardless of the absence or presence of normal visual inputs.

\section{Developmental change of the BDNF action}

Finally we tested whether the hypertrophic effect of BDNF on OD columns is seen in the matured cortex. For this we infused BDNF in the visual cortex of four adult cats in the same way as 
Figure 2. Difference in BDNF effects between a kitten and an adult cat: geniculocortical afferent termination in BDNF-treated cortex of a kitten (left) and an adult cat (right). Identifiers of the animals are indicated at the bottom left of $C$ and $F$. The BDNF infusion was initiated at postnatal day 38 in the kitten and lasted for $14 \mathrm{~d}$ in both animals. They were reared without visual deprivation. $A, D$, Examples of autoradiography of horizontal sections of the cortex. $B, E$, sections neighboring $A$ and $D$, respectively. Cortical regions perfused with BDNF were visualized as dark areas with immunohistochemical staining using anti-BDNF antibody. $C, F$, Superimposition of pairs of neighboring sections showing OD columns (green) and BDNF-infused regions (red). The superimposed area became yellow. Scale bar in $F, 2 \mathrm{~mm}$ (applies to $A-F$ ).
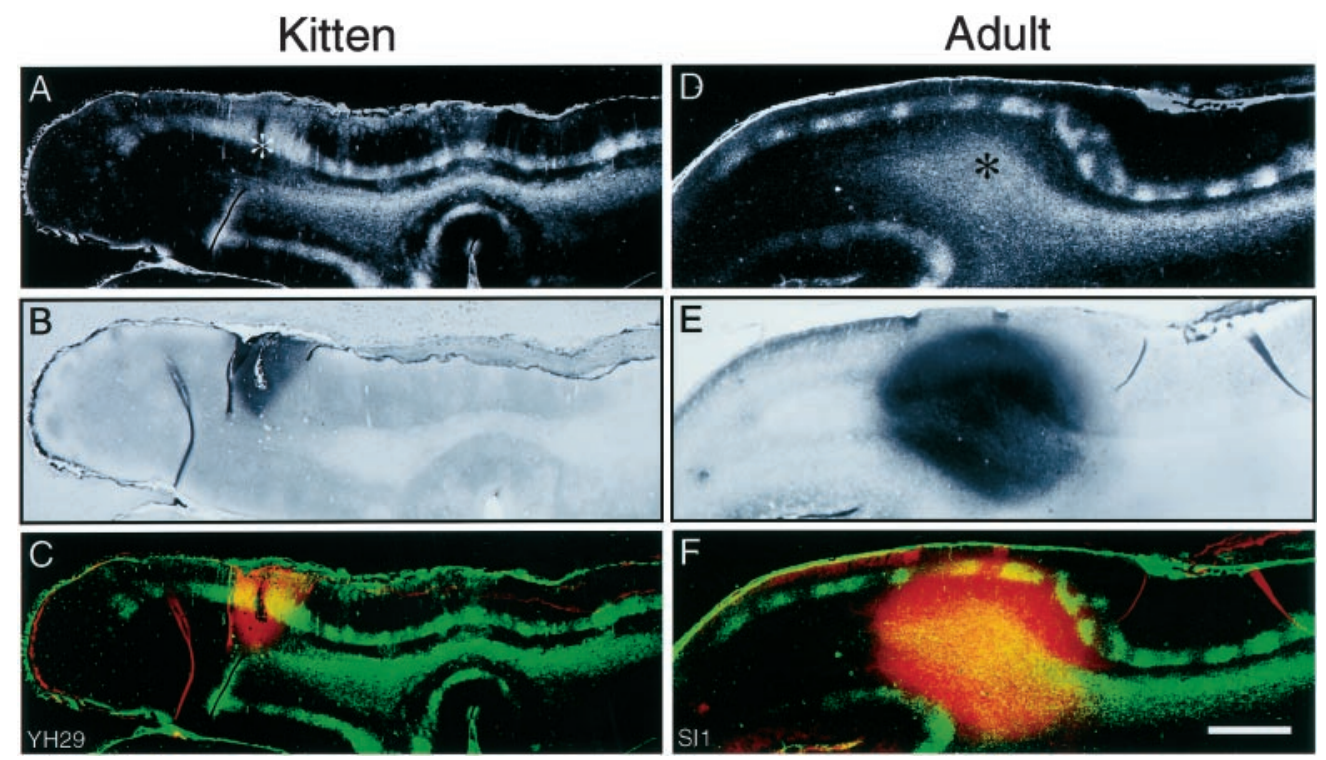

in the kittens. Figure 2 shows examples of horizontal sections of the cortex including the region inf used with BDNF from a kitten (Fig. 2A-C) and an adult cat (Fig. $2 D-F$ ). In autoradiography (Fig. $2 A, D$ ), layer IV of the visual cortex was labeled clearly in both animals. In the kitten, the labeling seems to be continuous in the area near the infusion site (asterisk) but to fluctuate almost regularly in the region far from that site (Fig. $2 A$ ). In the adult cat, on the other hand, a periodic fluctuation was clear even in the region closest to the infusion site (Fig. 2D), suggesting that the infused BDNF did not exert the expanding action on OD columns. There is a possibility, however, that the exogenously applied BDNF did not diffuse to layer IV at the effective concentration in the matured cortex. To test this possibility, we stained cortical sections immunohistochemically using anti-BDNF antibody and visualized the cortical region that had been perfused with BDNF. Immunohistochemical staining of the sections adjacent to those used for autoradiography revealed that BDNF spread well into layer IV of the visual cortex in the adult as well as in the kitten (Fig. 2E,B). Superimposition of the picture of autoradiography with that of immunohistochemistry confirmed that the periodic fluctuations of labeling which reflected OD columns were preserved well in the adult cortex even in the region perfused with BDNF (Fig. $2 F$ ). In the kitten, on the other hand, the continuously labeled region corresponded to that perfused with BDNF (Fig. 2C). We measured the spread of BDNF immunohistochemically in three kittens and three adult cats (including two kittens and one adult in addition to the three in Table 1). The extent of BDNF diffusion in the adults $(1.4,3.2$, and $5.5 \mathrm{~mm}$ in width) was not significantly different from that in the kittens (2.0, 2.3 , and $2.4 \mathrm{~mm} ; p=0.40, t$ test). Thus, it is unlikely that the failure for BDNF to expand OD columns in the matured cortex was attributable to the lack of BDNF. In other words, exogenous BDNF did not have the hypertrophic effect on matured OD columns in the visual cortex of adult cats.

To confirm the effect of BDNF on OD columns quantitatively, we measured the fraction of cortical territory occupied by labeled afferents (Fig. 3). In eight BDNF-treated kittens, the labeling occupied $100 \%$ of cortical area near the infusion site, whereas it made up $30-74 \%$ in the area far from the infusion site $(>4 \mathrm{~mm}$ anterior from the infusion site). The difference between these values for the near and far regions was statistically significant

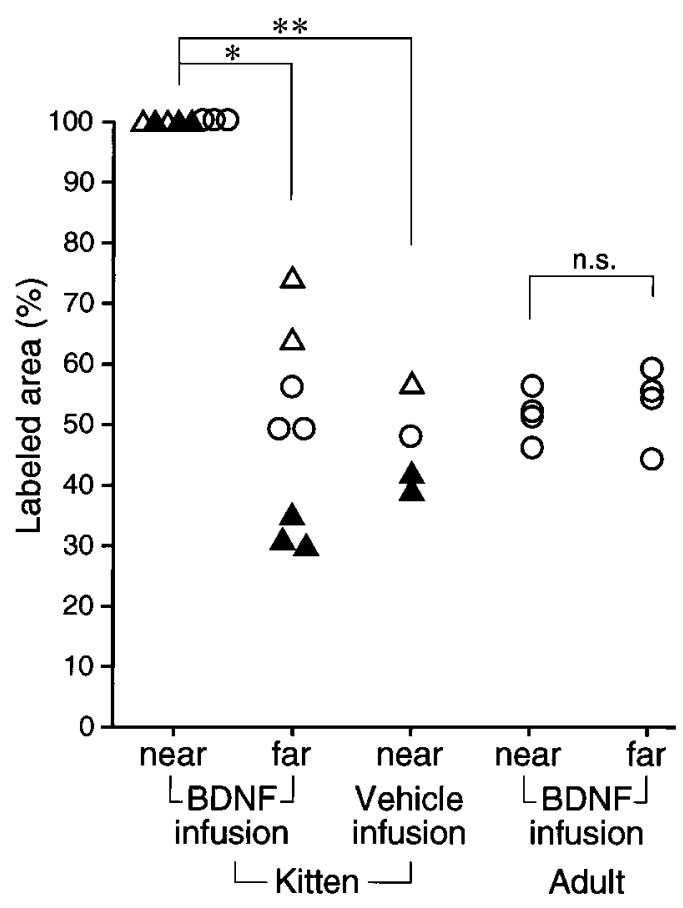

Figure 3. Quantitative analysis of the size of ocular dominance columns. The proportions of labeled area to a given cortical area were measured and plotted for each site. See Materials and Methods for details of measurements. Open triangles, filled triangles, and open circles represent values of the nondeprived eye columns, deprived eye columns, and columns of animals with normal vision, respectively. ${ }^{*} p<0.0001$ (paired $t$ test); ${ }^{* *} p<0.001$ (unpaired $t$ test); n.s., not significant.

( $p<0.0001$, paired $t$ test). In control hemispheres which were perfused with the vehicle solution only, the labeled area occupied $39-57 \%$ of the cortical area near the infusion site. This value is again significantly ( $p<0.001$, unpaired $t$ test) different from that of the near region in the BDNF-infused cortex. In the adult cats, the proportion of the labeled area in the region near the injection site was $46-56 \%$ and comparable to that in the far region (44$59 \%)$. The former value is not significantly different from the latter ( $p=0.28$, paired $t$ test). 


\section{DISCUSSION}

The present results have demonstrated that exogenously applied BDNF expands terminal areas of thalamocortical afferents in layer IV of the visual cortex irrespective of absence or presence of normal visual inputs, and that such an action of BDNF is seen only in kittens but not in adult cats. This expanding effect of BDNF was observed in the visual cortex from postnatal days 35-38 to 48-52, when OD columns have largely been formed (LeVay et al., 1978). Therefore, the lack of columnar structure around the BDNF infusion site observed in the present study could be interpreted as a result of promiscuous expansion of the already segregated afferents.

The expansion of OD columns by BDNF might be caused by some artifactual effects of exogenous BDNF rather than by its biological activity. If inf used BDNF had such a nonspecific effect, it should have caused an expansion of geniculocortical afferents in all directions in the cortex, and thus the spread of labeling should have been seen also in the supragranular and infragranular layers in addition to layer IV of the cortex. However, this was not the case. The labeling of geniculocortical afferents was largely confined within layer IV of the visual cortex even in the continuously labeled area, although a fraction of the labeling was observed also in the lower part of layer III (see Fig. 2A). This labeling in layer III may reflect an enhanced projection of LGN afferents to this layer, which exists in the normal cortex (LeVay and Gilbert, 1976), or another biological role of BDNF in establishing layerspecific projection between layers III and IV. Also, the present finding that OD columns were well preserved in the adult cortex even in the region perfused with BDNF further supports our view that the effect of exogenous BDNF observed in the present study reflects a biological role of BDNF that could be developmentally regulated. It remains to be confirmed, however, that endogenous BDNF exerts similar effects in the visual cortex, as observed in the present study with exogenous BDNF at a rather high concentration.

In MD kittens it was reported that an infusion of BDNF into the visual cortex shifted the eye preference of visual responses of cortical neurons rather to the deprived eye (Galuske et al., 1996), although NT-4/5 was reported to just block the expected shift to the nondeprived eye (Gillespie et al., 1996). The present findings seem not to be consistent with such a puzzling effect of BDNF in the former report, because BDNF exerted the expanding action on both deprived and nondeprived columns, and no significant difference was seen in the effects between the two kinds of columns in the present study. It is to be noted, however, that the reverse shift of OD of cortical neurons in the previous study was assessed with physiological methods such as optical imaging and extracellular recording of spike activity. Thus, the reverse shift might be a result of a modification of intracortical connections (McAllister et al., 1999) rather than that of geniculocortical connections.

The present results seem consistent with the hypothesis that BDNF plays a role in visual cortical plasticity as a trophic factor that is released from postsynaptic sites and acts on presynaptic terminals to expand the functional and morphological territory of afferents (Thoenen, 1995; Bonhoeffer, 1996; Katz and Shatz, 1996; Kinoshita et al., 1999), although we cannot completely exclude another possibility that infused BDNF might exert its sprouting effect on the dendrites of cortical neurons (McAllister et al., 1995) and deteriorate their visual responses and, consequently, desegregate ocular dominance columns indirectly through an interaction between cortical cells and afferents. If BDNF has such a trophic action on immature afferents, one can expect that the large amount of exogenously supplied BDNF would prevent input-deprived afferents from shrinking and further expand them in MD kittens. Also, if BDNF plays such a role in the monocular deprivation-induced change in the size of OD columns, the infusion of BDNF into the visual cortex should not have been effective in adult cats after cessation of the critical period. These are what we observed in the present study. Thus, the present results are consistent with the hypothesis that BDNF plays a role in activity-dependent plasticity of OD columns in the developing visual cortex as a trophic factor.

\section{REFERENCES}

Bonhoeffer T (1996) Neurotrophins and activity-dependent development of the neocortex. Curr Opin Neurobiol 6:119-126.

Cabelli RJ, Hohn A, Shatz CJ (1995) Inhibition of ocular dominance column formation by infusion of NT-4/5 or BDNF. Science 267:1662-1666.

Cabelli RJ, Shelton DL, Segal RA, Shatz CJ (1997) Blockade of endogenous ligands of TrkB inhibits formation of ocular dominance columns. Neuron 19:63-76.

Cellerino A, Maffei L (1996) The action of neurotrophins in the development and plasticity of the visual cortex. Prog Neurobiol 49:53-71.

Galuske RAW, Kim D-S, Castren E, Thoenen H, Singer W (1996) Brain-derived neurotrophic factor reverses experience-dependent synaptic modifications in kitten visual cortex. Eur J Neurosci 8:1554-1559.

Gillespie DC, Crair MC, Stryker MP (1996) NT-4/5 infusion alters plasticity and responses of cat primary visual cortex during the critical period. Soc Neurosci Abstr 22:276.

Hata Y, Stryker MP (1994) Control of thalamocortical afferent rearrangement by postsynaptic activity in developing visual cortex. Science 265:1732-1735.

Hata Y, Katsuyama N, Fukuda M, Ohshima M, Tsumoto T, Hatanaka H (1996) Brain-derived neurotrophic factor disrupts effects of monocular deprivation in kitten visual cortex. Soc Neurosci Abstr 22:1728.

Hubel DH, Wiesel TN (1970) The period of susceptibility of the physiological effects of unilateral eye closure in kittens. J Physiol (Lond) 206:419-436.

Katz LC, Shatz CJ (1996) Synaptic activity and the construction of cortical circuits. Science 274:1133-1138.

Kinoshita S, Yasuda H, Taniguchi N, Katoh-Semba R, Hatanaka H, Tsumoto T (1999) Brain-derived neurotrophic factor prevents lowfrequency inputs from inducing long-term depression in the developing visual cortex. J Neurosci 19:2122-2130.

LeVay S, Gilbert CD (1976) Laminar patterns of geniculocortical projections in the cat. Brain Res 113:1-19.

LeVay S, Stryker MP, Shatz CJ (1978) Ocular dominance columns and their development in layer IV of the cat's visual cortex. J Comp Neurol 179:223-244.

LeVay S, Wiesel TN, Hubel DH (1980) The development of ocular dominance columns in normal and visually deprived monkeys. J Comp Neurol 191:1-51.

McAllister AK, Katz LC, Lo DC (1995) Neurotrophins regulate dendritic growth in developing visual cortex. Neuron 15:791-803.

McAllister AK, Katz LC, Lo DC (1999) Neurotrophins and synaptic plasticity. Annu Rev Neurosci 22:295-318.

Purves D (1988) Body and brain: a trophic theory of neural connections. Cambridge, UK: Harvard UP.

Shatz CJ (1990) Impulse activity and the patterning of connections during CNS development. Neuron 5:745-756.

Shatz CJ, Stryker MP (1978) Ocular dominance in layer IV of the cat's visual cortex and the effects of monocular deprivation. J Physiol (Lond) 281:267-283.

Singer W (1995) Development and plasticity of cortical processing architecture. Science 270:758-764.

Stryker MP, Harris W (1986) Binocular impulse blockade prevents the formation of ocular dominance columns in cat visual cortex. J Neurosci 6:2117-2133.

Thoenen H (1995) Neurotrophins and neuronal plasticity. Science 270: 593-598. 